\title{
Genetic Myopathies Initially Diagnosed and Treated as Inflammatory Myopathy
}

\author{
Mark A. Tarnopolsky, Erin Hatcher, Rachel Shupak
}

\begin{abstract}
Objectives: Differentiating genetic myopathies from inflammatory myopathies can be challenging because of multiple overlapping clinical features. Examples are presented to highlight important clinical features that assist in the differentiation between the two. Methods: Clinical features including age at onset, history, pattern of weakness, serum creatine kinase activity, electromyography findings, and muscle biopsies are reported in six patients initially thought to have an inflammatory myopathy in whom the final diagnosis was a genetic myopathy. Results: All six patients met Bohan and Peter criteria for at least probable idiopathic polymyositis and were subsequently found to have a genetic myopathy (4DYSF, RYRl, and GNE). The key distinguishing clinical were minimal to no response to immunosuppression and atypical involvement of distal muscles in the majority of cases. Conclusions: Patients diagnosed with inflammatory myopathies should be reevaluated for the possibility of a genetic myopathy if they fail to respond to a course of diseasemodifying agents and/or there is atypical distal muscle involvement.
\end{abstract}

RÉSUMÉ: Cas de myopathies d'origine génétique diagnostiqués initialement et traités comme des cas de myopathies inflammatoires. Objectifs: Établir une distinction entre des cas de myopathies d'origine génétique et des cas de myopathies inflammatoires peut s'avérer difficile étant donné la multiplicité des aspects cliniques qui se chevauchent. Des exemples sont ici présentés afin de mettre en relief les aspects les plus susceptibles de permettre une distinction entre ces deux myopathies. Méthode: Les aspects cliniques incluent l'âge du patient au moment de l'apparition de la pathologie, l'histoire de cas, une tendance à la faiblesse musculaire, une activité sérique de la créatine kinase, ainsi que des résultats spécifiques à la suite d'un électromyogramme et de biopsies musculaires. Ces aspects ont été signalés chez six patients qu'on croyait au départ affectés par une myopathie inflammatoire et auxquels on a finalement transmis un diagnostic définitif de myopathie d'origine génétique. Résultats: Tous les six patients ont satisfait aux critères de Bohan et Peter, ce qui pourrait, à tout le moins, révéler la probabilité d'une polymyosite idiopathique. On a découvert par la suite qu'ils étaient atteints d'une myopathie d'origine génétique (gènes 4 DYSF, RYR1 et GNE). Dans la majorité des cas, les principaux signes cliniques se sont révélés être une faible, voire une absence, de réponse à l'immunosuppression et une atteinte atypique des muscles distaux. Conclusions: Les patients chez qui on a diagnostiqué des myopathies inflammatoires devraient être réévalués. Si ces derniers ne réagissent pas à un traitement immunomodulateur et/ou si l'on note chez eux une atteinte atypique des muscles distaux, il est en effet possible qu'ils soient atteints d'une myopathie d'origine génétique.

Keywords: dysferlinopathy, genetic myopathy, genetics, myositis, inflammatory diseases, inflammatory myopathy, myopathy doi:10.1017/cjn.2015.386

Can J Neurol Sci. 2016; 43: 381-384

The inflammatory myopathies (polymyositis [PM] and dermatomyositis $[\mathrm{DM}]$ ) are characterized by primary inflammation within skeletal muscle (PM, T cells invade nonnecrotic fibers; $\mathrm{DM}, \mathrm{B}$ cells in the peri- or endomysium, perifascicular atrophy, membrane attack complex, and often tubulo-reticular inclusions with electron microscopy). The prevalence of PM/DM is $~ 8.7 /$ 100,000 , and 10-year survival rate is $\sim 90 \%{ }^{1}$ PM and DM have traditionally been diagnosed using the Bohan and Peter criteria; proximal muscle weakness, elevated serum creatine kinase (CK) activity, electromyography (EMG) with active myositis and a muscle biopsy showing inflammation. ${ }^{2}$ The original criteria proposed that meeting all four criteria was sufficient for diagnosis of definite PM $(3 / 4=$ probable $)$, whereas three of the four plus a characteristic rash reflected definite DM $(2 / 4+$ rash $=$ probable).

There are several genetic myopathies in which secondary inflammation can occur including; calpainopathy, dysferlinopathy, fascioscapulohumeral muscular dystrophy, dystrophinopathy, and
LMNA-associated myopathy. ${ }^{3,4}$ Consequently, genetic myopathies represent a diagnostic challenge because they frequently show proximal weakness, elevated CK activity, and an abnormal EMG that can blur the distinction between inflammatory and noninflammatory myopathies. For example, a proportion of limb-girdle muscular dystrophy (LGMD) 2B/Miyoshi myopathy patients are initially misdiagnosed with $\mathrm{PM}^{4}$ The accurate diagnosis of a muscle disease is essential to the patient and clinician for therapy (i.e. supportive vs immunomodulation), genetic counseling (for the genetic myopathies), prognosis, and avoidance of iatrogenic side effects.

From McMaster University, Department of Pediatrics, Hamilton, Ontario, Canada (MAT, EH), St. Michaels Hospital, University of Toronto, Toronto, Ontario (RS).

Received March 3, 2015. Final Revisions Submitted September 24, 2015. Correspondence to: Mark Tarnopolsky, McMaster University, 1200 Main St. W., HSC-2H26, Hamilton, ON L8N 3 Z5 Canada

Email: tarnopol@mcmaster.ca 
The current paper describes six patients with inflammatory myopathy who received immunosuppressive therapy and were subsequently diagnosed with a genetic myopathy.

\section{Patients And Methods}

Each of the patients was referred to the McMaster University Neuromuscular and Neurometabolic clinic for evaluation of a myopathy and evaluated by the same investigator. Patients signed written informed consent for the muscle biopsy and ethics approval for writing the retrospective case series analysis was given by the Hamilton Health Sciences integrated Research Ethics Board. All patients had a neurological examination, CK, EMG, and a needle muscle biopsy (the latter in five of six patients). All genetic testing was completed using automated Sanger sequencing in commercial Clinical Laboratory Improvement Amendments-certified laboratories and all autosomal recessive mutations were confirmed to be in trans unless otherwise stated (patient 4).

Patient 1 presented with insidious proximal arm $>$ leg weakness at age 27 years. Investigations revealed elevated CK, myopathic EMG, and an open muscle biopsy of the quadriceps was reported as being consistent with "polymyositis." He was treated for more than 15 years with corticosteroids with minimal to no clinical or CK improvement (Table 1).

Patient 2 developed weakness over several months with proximal leg > arm weakness at age 17 years. CK was elevated and the EMG showed active myositis and muscle biopsy of the right deltoid was consistent with "polymyositis." She was treated with corticosteroids $(1 \mathrm{mg} / \mathrm{kg}$ ) for many months with neither clinical nor CK improvement (Table 1).

Patient 3 presented at age 15 years with difficulty keeping up in gym classes and proximal weakness. Open muscle biopsy of the Vastus lateralis showed "polymyositis." She was treated for 1 year with corticosteroids $(1 \mathrm{mg} / \mathrm{kg})$, several intravenous immunoglobulin G courses, and methotrexate. She had minimal strength improvement but CK remained $>5000$ IU $(\mathrm{n}<220)$ (Table 1$)$.

Patient 4 reported predominately proximal with some distal weakness (foot drop) progressing over a 1-year period, starting at age 20 years. CK was elevated, he had a myopathic EMG, and open muscle biopsy of the Vastus lateralis showed chronic inflammatory myopathy with perimysial inflammation and perivascular atrophy. He was treated with corticosteroids $(1.5 \mathrm{mg} / \mathrm{kg})$, azathioprine, methotrexate, intravenous immunoglobulin G, and pulse methylprednisolone with subjective worsening of his symptoms and no effect on CK over a 1-year period. All immunosuppression was stopped with no deterioration of clinical function or further elevation in CK (Table 1).

Patient 5 presented with fatigue, weakness, and myalgias in thigh muscles progressing over 1 year at age 28 years. Her CK activity was $7000 \mathrm{IU}$ and her EMG showing active myositis. She was started on methotrexate with no clinical response followed by prednisone, which halved the $\mathrm{CK}$ value but did not improve strength; after 6 months, azathioprine was added (Table 1).

Patient 6 was diagnosed with systemic lupus erythematosus (positive antinuclear antibodies and arthritis) at age 23 years. She has been treated with Plaquenil and prednisone. She noted proximal and distal leg weakness progressing slowly over the years that became clinically noticeable at age 42 years. CK was 400 to $700 \mathrm{IU}$, and she had no convincing benefit clinically or

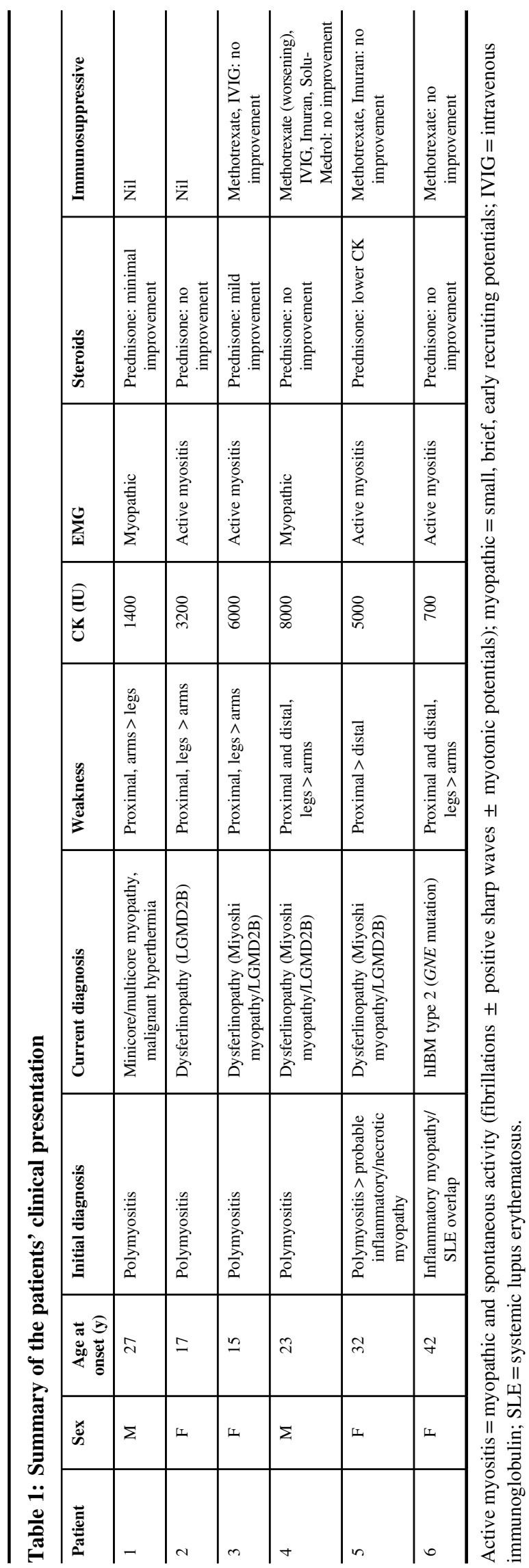




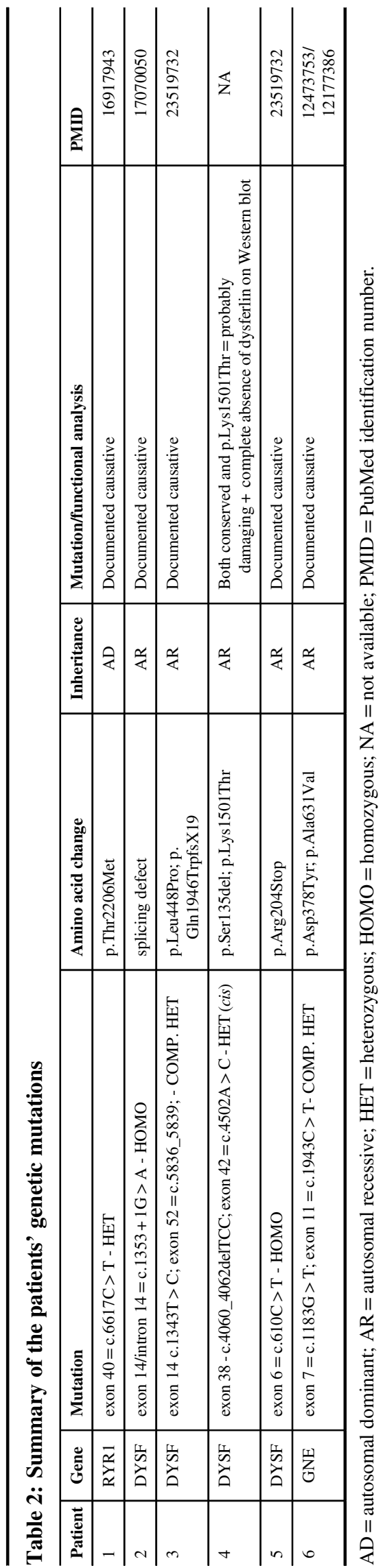

serologically to varying doses of prednisone for presumed overlap myositis (Table 1).

\section{Results \\ Patient 1}

Needle muscle biopsies were repeated 25 to 35 years after the diagnosis/initial biopsy showing dystrophic changes with no inflammation in both with multicores seen in the third biopsy. Gene mutation analysis for ryanodine receptor (RYRI) mutations revealed a mutation previously associated with malignant hyperthermia (Table 2).

\section{Patient 2}

Given that her brother developed similar features, her parents were consanguineous, and she had medial gastrocnemius atrophy, mutation analysis of the dysferlin $(D Y S F)$ gene showed a known homozygous mutation for LGMD2B/Miyoshi myopathy (Table 2).

\section{Patient 3}

When she was transferred to our center as an adult (at age 18 years), she was found to have profound medial gastrocnemius atrophy; a repeat muscle biopsy showed necrotic fibers, minimal fibrosis, some regenerating fibers, and no evidence of inflammation. She was tapered off methotrexate with no decline in clinical function, and $D Y S F$ mutation analysis revealed compound heterozygosity for documented LGMD2B/Miyoshi myopathy (Table 2). ${ }^{5}$

\section{Patient 4}

A needle muscle biopsy was completed of the Vastus lateralis showing muscular dystrophic change with necrosis and fibrosis with no inflammation. Because of the severe medial calf muscle atrophy and weakness, DYSF mutation analysis was done and showed two sequence variants that were highly conserved, with one sequence variant eliminating a serine residue and the second predicted to be "probably damaging" by polyphen-2. The two sequence variants were found to be in cis (present in asymptomatic father). Western blotting for dysferlin protein ${ }^{5}$ revealed complete absence in the presence of age-matched controls. Given the profound medial gastrocnemius atrophy, very high $\mathrm{CK}$, no response to corticosteroids, and the complete absence of dysferlin on Western blotting, a diagnosis of LGMD2B/Miyoshi was given (Table 2).

\section{Patient 5}

Upon reassessment, her CK was 5000 IU, EMG showed active myositis, and needle muscle biopsy showed necrotizing myopathy with neurogenic change, microangiopathy, and endomysial fibrosis. The finding of severe medial calf muscle atrophy, lack of response to immunosuppression, and the muscle biopsy showing no primary inflammation led to DYSF sequencing that confirmed a homozygous stop codon mutation, known to cause LGMD2B/ Miyoshi myopathy (Table 2). ${ }^{5}$

\section{Patient 6}

Neuromuscular clinic evaluation revealed distal $>$ proximal lower extremity weakness, CK of 700, EMG showed active myositis, and needle muscle biopsy of the right deltoid showed vacuolar myopathy with some denervation changes, minimal 
focal vasculitis, and type 1 fiber predominance. The distal weakness and biopsy prompted genetic analysis of $G N E$ showing compound heterozygosity for two known GNE mutations associated with hereditary inclusion body myopathy. Her sister had milder symptoms but some distal lower extremity weakness, hyper-CKemia, and was also found to have the same GNE mutations (Table 2).

\section{DisCUSSION}

The results of the current study highlight diagnostic challenges that arise in differentiating genetic from idiopathic inflammatory myopathies. A major issue is that nearly every muscular dystrophy shares three of the four defining features of PM (proximal weakness, elevated CK, myopathic EMG). Further complicating the differential diagnosis is that some muscular dystrophies also show secondary inflammation. ${ }^{3,4}$ A correct diagnosis is important to provide genetic counseling/prognosis and to avoid unnecessary treatment. In addition to the well-known side effects of immunosuppressive therapy (e.g. infections, weight gain, dysglycemia, blood dyscrasias), there have been case reports of a more rapid deterioration with the use of corticosteroids in patients with LGMD2B/Miyoshi myopathy, further emphasizing the need to arrive at an accurate diagnosis before initiating therapy.

LGMD2B/Miyoshi myopathy is probably the most common myopathy to be confused with PM given the inflammatory changes often seen in the biopsy, high CK, proximal weakness, and similar EMG changes. ${ }^{4}$ We also support these findings in that $66 \%$ of our patients had DYSF mutations or one mutation and a severe loss of DYSF protein. It is felt that DYSF deficiency directly affects inflammatory cells (monocytes) that in combination with muscle damage, leads to an inflammatory cascade that activates and perpetuates the dystrophic process. Another issue that may contribute to the misdiagnosis of LGMD2B/Miyoshi myopathy is the rapid progression of the disease seen in $\sim 25 \%$ of patients in which previously subclinical weakness progresses rapidly and appears to be an inflammatory myopathy. ${ }^{4}$ A positive family history of weakness or myopathy is one important clue to a genetic diagnosis, albeit some disorders can have variable penetrance and autosomal recessive disorders often appear to be sporadic in small, nonconsanguineous families. Although dermatomyositis commonly presents in childhood, it is distinctly unusual for polymyositis or overlap myositis to appear in people $<20$ years of age.

We have also found that the finding of severe medial gastrocnemius muscle atrophy/weakness (tested in the standing position) is a valuable clinical finding to suggest LGMD2B/ Miyoshi myopathy, and this was the key feature in each of our four DYSF patients. Indeed, if there is significant weakness of the distal lower extremities (five of six in our series) in a patient with suspected inflammatory myopathy, it is important to consider evaluation for a genetic myopathy. Part of the confusion with inflammatory myopathies is that the distal myopathies often have some degree of proximal weakness as seen in five of our patients with distal myopathies. In our experience, all of the 20 patients with documented DYSF mutations seen in our clinic have both proximal (LGMD2B) and disproportionate plantar flexor (Miyoshi myopathy) weakness. It is important to specifically test the plantar flexors in the standing position for often the medial calf atrophy is not apparent in the supine position and the strength appears normal.

\section{CONCLUSION}

Overall, we find that the original Bohan and Peter criteria with careful evaluation of the distal lower extremity muscles are helpful in differentiating the genetic from inflammatory myopathies; however, it is important to only assign a definitive inflammatory myopathy diagnosis to patients with: (1) proximal with minimal to no distal foot weakness; (2) EMG showing both active (positive sharp waves, fibrillations) and myopathic changes (small, brief, early recruiting potentials); and (3) characteristic muscle biopsy findings. The muscle biopsy can occasionally be confusing for T cells and major histocompatibility complex I upregulation can also be seen in LGMD2B/Miyoshi myopathy ${ }^{3}$; however, the medial gastrocnemius atrophy/weakness provides an important diagnostic clue. In rare cases that strictly meet all of the aforementioned criteria and fail to respond to immunosuppression, it is important to reevaluate the patient and consider testing for genetic myopathies.

\section{Disclosures}

MAT has received speaker honoraria from Prevention Genetics (2014). MAT has filed a patent for the use of exosomemRNA based therapies in hereditary myopathies. EH and RS do not have anything to disclose. The authors of this paper have not received any financial support or other benefits from commercial sources for the work reported on in this manuscript, nor do they have any other financial interests which could create a potential conflict with regard to the work.

\section{REFERENCES}

1. Taborda AL, Azevedo P, Isenberg DA. Retrospective analysis of the outcome of patients with idiopathic inflammatory myopathy: a long-term follow-up study. Clin Exp Rheumatol. 2014;32: 188-93.

2. Bohan A, Peter JB. Polymyositis and dermatomyositis (second of two parts). N Engl J Med. 1975;292:403-7.

3. Fanin M, Angelini C. Muscle pathology in dysferlin deficiency. Neuropathol Applied Neurobiol. 2002;28:461-70.

4. Nguyen K, Bassez G, Krahn M, et al. Phenotypic study in 40 patients with dysferlin gene mutations: high frequency of atypical phenotypes. Arch Neurol. 2007;64:1176-82.

5. Nilsson MI, Laureano ML, Saeed M, Tarnopolsky MA. Dysferlin aggregation in limb-girdle muscular dystrophy type 2B/Miyoshi myopathy necessitates mutational screen for diagnosis [corrected]. Muscle Nerve. 2013;47:740-7. 